\title{
Socio-demographic influences on the prevalence of intestinal parasitic infections among workers in Qatar
}

\author{
Nadin Younes ${ }^{1}$, Jerzy M. Behnke ${ }^{2}$, Ahmed Ismail ${ }^{3}$ and Marawan A. Abu-Madi ${ }^{*}$
}

\begin{abstract}
Background: The rapid growth of Qatar in the last two decades has been associated with an enormous expansion of building programs in its cities and in the provision of new service industries. This in turn has attracted a large influx of immigrant workers seeking employment in jobs associated with food handling, domestic service, and the building industry. Many of these immigrants come from countries in the tropics and subtropics where intestinal parasitic infections are common. In this study, we explored the environmental and socio-demographic characteristics of immigrant workers in Doha Qatar, which might explain the persistence of the parasites that they harbor.

Methodology: This cross-sectional survey was conducted among 2486 newly arrived immigrant workers and those who visited Qatar previously during the period 2012-2014. Through questionnaires and census data, we characterized the socio-demographic conditions at an individual, family, and neighborhood levels.

Results: Overall, the prevalence of combined protozoan infection was $11.7 \%$ and that of helminth was $7.0 \%$. Combined protozoan infections were significantly associated with immigrant workers arriving in Doha for the first time. In univariate log-linear statistical models fitted in phase 1 of the analysis, significant associations were observed between the prevalence of combined protozoan infections and personal and familial factors that included religion, the level of education of subjects, both parents' educational levels and their jobs, and the number of siblings. Furthermore, environmental effects on the prevalence of protozoan infections including the country of origin, the floor of the house, toilet type, household content index, provision of household water, farming background showed strong associations with protozoan infections. However, in phase 2, multifactorial binary logistic generalized linear models focusing only on the significant effects identified in phase 1, showed that only five factors retained significance (age class, floor of the house, household contents index, father's education, and the number of siblings). The only factors that had a significant effect on the prevalence of helminth infections were the subjects' age class and the mother's educational level.

Conclusions: The prevalence of intestinal protozoan parasites among immigrant workers in Qatar is clearly multifactorial in origin determined by key familial relationships of subjects and also the environment, in which the subjects lived prior to their arrival in Qatar. Moreover, our results suggest that screening protocols for applicants for visas/work permits need to be revised giving more careful attention to the intestinal protozoan infections that potential immigrant workers may harbor.
\end{abstract}

Keywords: Helminth, Protozoa, Immigrant workers, Prevalenc, Qatar

*Correspondence: abumadi@qu.edu.qa

1 Biomedical Science Department, College of Health Sciences, Biomedical

Research Center, Qatar University, P.O. Box 2713, Doha, Qatar

Full list of author information is available at the end of the article permits use, sharing, adaptation, distribution and reproduction in any medium or format, as long as you give appropriate credit to the original author(s) and the source, provide a link to the Creative Commons licence, and indicate if changes were made. The images or other third party material in this article are included in the article's Creative Commons licence, unless indicated otherwise in a credit line to the material. If material is not included in the article's Creative Commons licence and your intended use is not permitted by statutory regulation or exceeds the permitted use, you will need to obtain permission directly from the copyright holder. To view a copy of this licence, visit http://creativecommons.org/licenses/by/4.0/. The Creative Commons Public Domain Dedication waiver (http://creativeco mmons.org/publicdomain/zero/1.0/) applies to the data made available in this article, unless otherwise stated in a credit line to the data. 


\section{Introduction}

Intestinal parasitic infections continue to be a major public health problem especially in low- and middle-income populations $[1,2]$, and one of the major causes of morbidity and mortality particularly in developing countries [3]. Prevalence is known to be closely related to the educational levels of subjects, environmental factors, sanitary conditions, socio-economic status, inadequate medical care, and lack of access to safe drinking water supplies [4-6]. Studies have also shown that social and economic contexts are important determinants of human health, including diseases caused by parasitic organisms [2].

Asymptomatic infected food handlers and housemaids are a potential source of infection for many intestinal parasites and other enteropathogenic infectious agents $[7,8]$. Economic migrants from these groups, but including also those engaged in other jobs such as laborers and drivers, who harbor parasitic infections carry them to the countries in which they settle unless they are treated before arrival [9]. The transmission of parasites occurs directly or indirectly through food, water, or hands, reinforcing the importance of fecal-oral and human-to-human transmission modes [10]. Thus, food handlers with poor personal hygiene and inadequate knowledge of food safety could be the source of foodborne pathogens and may be implicated in the transmission of many infections to the local community, posing a particular infection risk to the public $[8,11,12]$.

Recently, the Arabian Gulf region has seen enormous progress in the living standards of its inhabitants and this has attracted immigrant workers seeking work from around the globe. In Qatar, most of the immigrant workers who work as drivers, food handlers, housemaids, and child/early care assistants come from a background of modest socio-economic living standards in their countries of origin. Many are from areas where protozoan infections are endemic, and where there is no or inadequate access to medical care services. It is important therefore to alert both the immigrant workers and the local communities in which they have settled about the risks of these contagious diseases, particularly the factors that facilitate parasite transmission, to limit the spread of the infectious agents.

In an earlier paper on this dataset, we focused on the extrinsic (nationality and region of origin) and intrinsic factors (sex and age) that affected both combined helminth infections (all 7 species combined and treated as one taxon) and combined protozoan infections (all 8 species combined and treated as one taxon) among recently arrived immigrant workers to Qatar [13]. Age, sex, and nationality were all recorded at the Medical Commission as part of the routine clinical inspection of applicants for work permits. In the previous study, we found that only an age effect was significant for combined helminth infections. There was no difference in the prevalence of combined helminths between subjects from different regions of origin and no difference in prevalence between the sexes. For combined protozoan infections both the regional and age effects were significant but, as with helminths, there was no difference in prevalence between the sexes.

In this study, we build on the earlier published analysis, seeking socio-demographic factors that might provide further explanations for variation in the prevalence of protozoan and helminth infections among immigrant workers. The factors used in this analysis were derived from a detailed survey instrument, that was completed with the help of a trained translator, by randomly chosen subjects at the initial presentation.

\section{Methods}

\section{Study population and sample collection}

We conducted a cross-sectional study on the prevalence of intestinal parasites in Qatar among immigrant workers in certain jobs, which are white collar workers (a professional or educated worker in office positions), blue collar worker (physical/manual labourers), and pink collar workers (care oriented and typically performed by women), food handlers, and housemaids. Briefly, 2486 subjects from 24 countries were screened during the period 2012-2014. Stool samples were collected from randomly selected individuals during routine health examinations, soon after arrival in the country when they reported to the Medical Commission in order to obtain work permits. The subjects were allocated to four age classes [Age class 1 (16-22 years, $n=303)$; Age class 2 (23-29 years, $n=856$ ); Age class 3 ( $30-37$ years, $n=823$ ); and Age class $4(38-58$ years, $n=504)]$, and to four regions of origin [Eastern Asia $(n=936)$; Western Asia $(n=1289)$; Northern and Saharan Africa $(n=138)$; and sub-Saharan Africa $(n=123)$, as described by Abu Madi et al. [14].

\section{Stool examination}

Stool examination was carried out in a safety cabinet, where stool specimens were preserved in an ecofix preservative vial (Meridin Biosciences, Inc., Cincinnati, $\mathrm{OH})$. The contents were stirred with fine clean disposable wooden sticks to remove large clumps and mixed vigorously by vortex to homogenize the sample. To ensure adequate fixation of the homogenized stool, the sample was kept for half an hour at room temperature. The preserved specimen was mixed by vortex and filtered through a macro-con filtration unit for the removal of bulky debris. After filtration, 10\% formalin and ethyl acetate were added, the sample was centrifuged for $10 \mathrm{~min}$ 
at $3000 \mathrm{rpm}$, and the fluid containing diethyl ether and formalin was discarded. The pellet was resuspended by agitation, poured onto a microscope slide containing one drop of iodine, and examined microscopically for the presence/absence of parasite eggs/cysts and to enable identification of parasites in positive samples [15].

\section{Socio-demographic data collection and analysis}

A socio-demographic questionnaire was also completed for all participants by trained interviewers. Information obtained at interviews was first recorded on hard copies of printed pro-formas of the questionnaire. Subsequently, data were entered into an Excel workbook and subjected to quality control procedures. Numerical data were then imported into SPSS version 23 for analysis and were treated initially under two headings: personal and familial characteristics, and environmental factors reflecting aspects of the living conditions in the respondents' home villages, towns or cities. Each recorded factor was divided into a range of levels. Some were simple binary entries (e.g. yes or no, and coded as 1 and 0 respectively), others comprised more levels and were given numerical nominal coding, and finally, it was possible in some cases to scale the values provided by respondents (e.g. no of siblings).

Personal and familial factors included; immigration status, religion, education, job/profession, monthly income, number of siblings, father's education, father's occupation/profession, mother's education, mother's occupation/profession.

Environmental factors including the ownership of the house, number of people sharing a house, number of rooms, house construction, floor of the house, toilet, provision of household water, whether the subject was a farmer, and whether the subject owned any domestic animals and if so how many different species. The number of animal species was based on a choice from dog, goat, cow, cat, chicken, and other (examples specified by 27 subjects included birds, ducks, buffalo, ox, sheep, pigs) and 1 point was given for each species. The household contents index was based on 1 point for each of the following: gas or electricity cooker, microwave oven, fridge, television, radio, computer, internet access, shower, bath, and car.

\section{Statistical analysis}

Analysis of data was undertaken in two phases because of the number of potential explanatory factors recorded in the questionnaire. First, univariate log-linear models were fitted with each factor in turn and INFECTION (either combined helminths or combined protozoan infections, each at 2 levels, present or absent), as described elsewhere [16]. Then, the significant factors from the initial phase were selected, and multifactorial generalized linear models (GLMs) were fitted with a binary log link in SPSS 23, incorporating all the main effects and all relevant 2-way interactions. Because of the number of factors involved, this second phase was conducted in three separate stages. First, model 1 was fitted with all the significant familial factors from phase 1 , and then a second model (model 2) was fitted with the environmental factors, and in each case also including age class and region of origin of subjects as these had been shown earlier to have had a significant effect on INFECTION [14]. In a third stage, the significant factors from models 1 and 2 were included in model 3 that also incorporated age class and region of origin. Model simplification was by the backward selection, deleting the least significant interaction in turn at each successive cycle, until only significant 2-way interactions, and relevant main effects remained. Significance was based on the Wald $\chi^{2}$ output of the minimum sufficient model thus generated. The final minimum sufficient model was also tested by multifactorial $\log$-linear analysis, to confirm parameter estimates.

Data are reported as prevalence values (percentage of infected subjects in relevant factor levels) with 95\% confidence limits in parenthesis. We provide also odds ratios $+95 \%$ confidence limits for levels within each factor, using one level as the reference point in each case. Relationships between the prevalence of infection and levels within specific factors that showed a directional trend (meaningful increase across levels e.g. no of siblings in the family) were examined by the non-parametric Spearman's test in SPSS 23, and $r s$ is given. $P$-values less than 0.05 were considered to indicate statistical significance.

\section{Results}

A total of 2486 samples (male $=1351,54.3 \%$ and female $=1135,45.7 \%)$ were included in the study. The overall prevalence of infections with combined helminths; (the seven helminth taxa) was $7.0 \%$ (95\% CL: 6.03-8.05\%) and with combined protozoan infections (the eight protozoan taxa) was $11.7 \%$ (95\% CL:10.40$12.93 \%)$. The prevalence of each of the individual species that were detected is shown in Additional file 1: Table S1. The prevalence of combined helminths and combined protozoa at each level of the personal, familial, and environmental variables that were recorded are given in Tables 1 and 2 .

\section{Personal and familial characteristics}

Interestingly, based on univariate analysis, no significant difference in the prevalence of combined helminths between the newly and previously arrived immigrant workers was observed (Table 1). No significant difference 
in the prevalence of combined helminth infections was found between different religions, education status, job profession, income status, number of siblings, father's education, father's occupation, and mother's occupation. The only factor that was found to have a significant effect on the prevalence of combined helminth infection was the mother's educational level. However, unexpectedly, the prevalence of combined helminth infection was the highest among those whose mothers had only experienced elementary school and those whose mothers went on to finish universities (Table 1). Whereas, the lowest prevalence of helminth infection was observed among immigrants whose mothers experienced only intermediate and high schools.

In contrast to the helminths, first-time arrivals in Qatar had a significantly higher prevalence of combined protozoan infections than those who had visited previously. The prevalence of combined protozoa was affected also by religion (highest among Hindu, lowest among Buddhists), personal education (highest among those with none, lowest among graduates), number of siblings, father's educational level (highest if none, lowest if he had attended at least high school), father's job/profession (highest if none, lowest if a white collar worker) and mother's education (highest if none, lowest if she had attended at least high school). The Mother's job had borderline significance (highest in those with no job).

Even though the relationship between the presence/ absence of helminths and the number of siblings in the family was not significant (Table 1), there was a significant positive correlation between prevalence and the number of siblings $\left(r_{s}=0.76, n=8, P=0.028\right.$; see Fig. 1$)$. Moreover, in comparison to the families with no children, the odds ratios for all those with children were significantly higher. The prevalence of combined protozoa varied significantly among the different levels corresponding to the number of siblings, but there was no directional trend (highest among those with 6, but surprisingly lowest among those with 5), not surprisingly the correlation between prevalence and no of siblings was not significant $\left(r_{s}=0.13, n=8, P=0.76\right)$.

\section{Environmental characteristics in the country of origin}

The only environmental factor that showed a significant impact on the prevalence of combined helminths in the univariate analysis was the household contents index, but no clear trend was found in relation to an increasing index that might have reflected increasing affluence (Table 2). Nevertheless, several environmental factors were significantly associated with the prevalence of combined protozoan infections; number of rooms in the house (highest if the house contained 4 rooms, lowest if the house contained 5 to 25 rooms, floor of the house (highest if the house floor is soil, lowest if wooden floor or hard natural surface), type of toilet facility (highest in those living in houses with pit latrines), household contents index (surprisingly highest value among those with an index of 10, but excepting this level, highest values were the bottom end of the index), household water supply (highest for those using river water, but also surprisingly high for those with internal water supplies, although low if using bottled water and also a covered well). There were no infections among those using shared taps, but the sample size for this group was small. Prevalence of combined protozoan infections was surprisingly higher among non-farmers compared with farmers.

\section{Controlling for combinations of socio-demographic and environmental factors in analysis of combined helminth infections}

The significant factors identified in the first phase as affecting the prevalence of combined helminth infections were age class of subjects, the number of siblings, mother's education, and household contents index. Age class of subjects was reported as a significant factor in this data-set in our earlier publication [14]. Following backward selection and model simplification, the minimum sufficient GLM model comprised only the main effects of age class (Wald $\chi_{3}^{2}=12.2, P=0.007$ ) and a weak effect of the mother's education level (Wald $\chi_{3}^{2}=9.7, P=0.046$ ) as factors influencing the prevalence of helminths in this dataset. However log-linear analysis identified also a weak significant interaction between age class, and the mother's education level $\left(\chi_{12}^{2}=23.6, P=0.023\right)$, and this is presented in Table 3 . The highest prevalence of combined helminth infections in age classes 1,3 , and 4 were among participants whose mothers had undergone tertiary level education, whereas, among participants in age class 2, the highest prevalence was among those whose mothers had no education.

\section{Controlling for combinations of socio-demographic and environmental factors in analysis of combined protozoan infections}

We fitted a GLM that comprised all the significant personal and familial factors identified in Table 1 (9 factors, comprising immigration, religion, education, father's education, father's occupation, number of siblings and mother's education, plus the region of origin and age class) as main effects and their 2-way interactions. The minimum sufficient model (model 1) was age (Wald $\left.\chi_{3}^{2}=13.0, P=0.005\right)$ as expected from Abu-Madi et al. [14], and then the number of siblings (Wald $\chi_{7}^{2}=15.6$, $P=0.03$ ) and father's education (Wald $\chi_{4}^{2}=32.5$, 
Table 1. The prevalence of helminths and protozoan infections of subjects in relation to personal and familial characteristics of subjects

\begin{tabular}{|c|c|c|c|c|c|c|}
\hline & \multirow[t]{2}{*}{$N$} & \multirow[t]{2}{*}{$\%$} & \multicolumn{2}{|l|}{ Combined helminths } & \multicolumn{2}{|l|}{ Combined protozoa } \\
\hline & & & Prevalence (95\% CL) & Odds ratio (95\% CL) & Prevalence (95\% CL) & Odds ratio $(95 \% \mathrm{CL})$ \\
\hline \multicolumn{7}{|l|}{ Immigration } \\
\hline First arrival & 2304 & 92.6 & $7.2(6.15-8.26)$ & 1 & $12.1(10.75-13.40)$ & 1 \\
\hline Has previously visited & 182 & 7.4 & $4.9(2.09-10.83)$ & $0.670(0.337-1.334)$ & $6.6(3.20-12.84)$ & $\mathbf{0 . 5 1 4}(0.283-0.936)$ \\
\hline Statistical test & & & $\chi_{1}^{2}=1.45, P=0.23$ & & $X_{1}^{2}=5.64, P=0.018$ & \\
\hline \multicolumn{7}{|l|}{ Religion } \\
\hline Buddhist & 76 & 3.1 & $5.3(1.73-13.68)$ & 1 & $5.3(1.73-13.68)$ & 1 \\
\hline Christian & 695 & 28 & $5.9(4.37-7.84)$ & $1.128(0.393-3.241)$ & $9.6(7.67-12.00)$ & $1.920(0.680-5.422)$ \\
\hline Hindu & 532 & 21.4 & $6.6(5.13-8.35)$ & $1.268(0.438-3.672)$ & $15.0(12.87-17.45)$ & $3.186(1.132-8.964)$ \\
\hline Muslim & 1178 & 47.5 & $8.1(6.53-9.86)$ & $1.579(0.565-4.416)$ & $11.7(9.88-13.55)$ & $2.388(0.859-6.640)$ \\
\hline Sikh & $5^{\mathrm{a}}$ & - & $0(0-50.00)$ & - & $20.0(1.03-65.74)$ & - \\
\hline Statistical test & & & $\chi_{3}^{2}=3.84, P=0.28$ & & $X_{3}^{2}=12.07, P=0.007$ & \\
\hline \multicolumn{7}{|l|}{ Education } \\
\hline None & 566 & 22.8 & $7.6(6.00-9.52)$ & 1 & $15.2(12.97-17.69)$ & 1 \\
\hline Elementary school only & 819 & 32.9 & $7.6(5.70-9.94)$ & $0.996(0.665-1.493)$ & $13.1(10.61-15.96)$ & $0.839(0.617-1.140)$ \\
\hline Up to intermediate school & 287 & 11.5 & $7.0(4.73-10.07)$ & $0.911(0.525-1.580)$ & $11.5(8.55-15.22)$ & $0.725(0.472-1.114)$ \\
\hline Up to high school & 575 & 23.1 & $6.1(4.65-7.86)$ & $0.788(0.497-1.251)$ & $9.4(7.60-11.50)$ & $0.578(0.403-0.831)$ \\
\hline Graduate/postgraduate & 239 & 9.6 & $6.3(4.30-9.00)$ & $0.814(0.443-1.496)$ & $4.2(2.63-6.50)$ & $0.244(0.124-0.478)$ \\
\hline Statistical test & & & $\chi_{4}^{2}=1.66, P=0.80$ & & $X_{4}^{2}=27.6, P<0.001$ & \\
\hline \multicolumn{7}{|l|}{ Job/profession ${ }^{b}$} \\
\hline Blue collar worker & 870 & 35 & $8.0(6.07-10.58)$ & 1 & $13.0(10.47-15.99)$ & 1 \\
\hline Pink collar worker & 167 & 6.7 & $4.8(2.08-10.25)$ & $0.575(0.271-1.219)$ & $8.4(4.46-14.88)$ & $\mathbf{0 . 6 1 3}(0.343-1.097)$ \\
\hline White collar worker & 67 & 2.7 & $6.0(2.30-13.81)$ & $0.726(0.257-2.052)$ & $7.5(3.18-15.80)$ & $0.540(0.213-1.373)$ \\
\hline Housemaid & 1231 & 49.5 & $7.0(5.59-8.63)$ & $0.858(0.68-1.192)$ & $11.4(9.60-13.15)$ & $0.860(0.660-1.120)$ \\
\hline Food handler & 151 & 6.1 & $4.6(2.08-9.66)$ & $0.556(0.250-1.233)$ & $11.9(7.38-18.49)$ & $0.907(0.533-1.541)$ \\
\hline Statistical test & & & $\chi_{4}^{2}=4.37, P=0.36$ & & $\chi_{4}^{2}=4.75, P=0.31$ & \\
\hline \multicolumn{7}{|l|}{ Monthly income } \\
\hline 600-999 QR & 1196 & 48.1 & $7.6(6.13-9.34)$ & 1 & $11.0(9.26-12.81)$ & 1 \\
\hline 1000-1499 QR & 783 & 31.5 & $7.2(5.37-9.40)$ & $0.935(0.662-1.322)$ & $12.4(10.05-15.16)$ & $1.140(0.862-1.507)$ \\
\hline 1500-2999 QR & 413 & 16.7 & $6.1(3.67-9.75)$ & $0.782(0.495-1.236)$ & $12.8(9.18-17.61)$ & $1.187(0.844-1.668)$ \\
\hline$>2999$ QR & 94 & 3.8 & $3.2(0.53-12.00)$ & $0.400(0.124-1.290)$ & $8.5(3.23-19.10)$ & $0.750(0.355-1.582)$ \\
\hline Statistical test & & & $\chi_{3}^{2}=3.87, P=0.28$ & & $\chi_{3}^{2}=2.38, P=0.50$ & \\
\hline \multicolumn{7}{|l|}{ No. of siblings } \\
\hline 0 & 82 & 3.3 & $3.7(0.82-11.79)$ & 1 & $11.0(5.11-21.35)$ & 1 \\
\hline 1 & 240 & 9.7 & $5.8(3.94-8.50)$ & $1.631(0.457-5.826)$ & $12.1(9.28-15.53)$ & $1.115(0.504-2.466)$ \\
\hline 2 & 431 & 17.3 & $6.7(4.14-10.67)$ & $1.900(0.565-6.389)$ & $12.1(8.42-16.81)$ & $1.113(0.525-2.357)$ \\
\hline 3 & 439 & 17.7 & $5.7(3.34-9.45)$ & $1.590(0.469-5.394)$ & $9.1(5.99-13.62)$ & $0.813(0.378-1.747)$ \\
\hline 4 & 401 & 16.1 & $8.2(5.43-12.32)$ & $2.361(0.707-7.893)$ & $14.5(10.60-19.30)$ & $1.372(0.650-2.893)$ \\
\hline 5 & 309 & 12.4 & $8.1(5.61-11.57)$ & $2.318(0.682-7.877)$ & $7.8(5.34-11.18)$ & $0.683(0.304-1.532)$ \\
\hline 6 & 221 & 8.9 & $8.6(6.34-11.57)$ & $2.477(0.713-8.603)$ & $16.3(13.13-19.96)$ & $1.578(0.724-3.440)$ \\
\hline$>6^{c}$ & 363 & 14.6 & $7.4(4.90-11.12)$ & $2.116(0.626-7.151)$ & $11.6(8.28-15.82)$ & $1.061(0.495-2.277)$ \\
\hline Statistical test & & & $\chi_{7}^{2}=5.8, P=0.56$ & & $x_{7}^{2}=15.2, P=0.033$ & \\
\hline \multicolumn{7}{|l|}{ Father's education } \\
\hline None & 1550 & 62.3 & $7.1(5.82-8.38)$ & 1 & $14.6(12.82-16.34)$ & 1 \\
\hline Elementary school only & 495 & 19.9 & $8.1(5.02-12.75)$ & 1.151 (0.789-1.678) & $7.3(4.38-11.74)$ & $\mathbf{0 . 4 5 9}(0.318-0.663)$ \\
\hline Up to intermediate school & 109 & 4.4 & $9.2(5.71-14.32)$ & $1.322(0.671-2.607)$ & $7.3(4.33-12.08$ & $0.464(0.223-0.966)$ \\
\hline Up to high school & 225 & 9.1 & $4.0(2.51-6.20)$ & $0.545(0.272-1.092)$ & $5.8(3.93-8.34)$ & $0.359(0.202-0.640)$ \\
\hline Graduate/post-graduate & 107 & 4.3 & $5.6(3.07-9.85)$ & $0.778(0.334-1.812)$ & $6.5(3.76-11.02)$ & $\mathbf{0 . 4 1 0}(0.188-0.894)$ \\
\hline
\end{tabular}


Table 1. (continued)

\begin{tabular}{|c|c|c|c|c|c|c|}
\hline & \multirow[t]{2}{*}{$N$} & \multirow[t]{2}{*}{$\%$} & \multicolumn{2}{|l|}{ Combined helminths } & \multicolumn{2}{|l|}{ Combined protozoa } \\
\hline & & & Prevalence (95\% CL) & Odds ratio (95\% CL) & Prevalence (95\% CL) & Odds ratio $(95 \% \mathrm{CL})$ \\
\hline Statistical test & & & $\chi_{4}^{2}=5.57, P=0.23$ & & $X_{4}^{2}=37.0, P<0.001$ & \\
\hline \multicolumn{7}{|l|}{ Father's occupation/profession } \\
\hline None & 1160 & 46.9 & $6.8(5.39-8.49)$ & 1 & $12.6(10.68-14.50)$ & 1 \\
\hline Blue collar worker & 1106 & 44.7 & $7.4(5.90-9.20)$ & $1.096(0.795-1.510)$ & $11.9(10.02-13.85)$ & $0.941(0.732-1.210)$ \\
\hline White collar worker & 207 & 8.4 & $6.3(4.38-8.82)$ & $0.917(0.500-1.681)$ & $5.3(3.61-7.69)$ & $0.390(0.207-0.733)$ \\
\hline Unknown ${ }^{d}$ & 13 & & & & & \\
\hline Statistical test & & & $\chi_{2}^{2}=0.52, P=0.77$ & & $X_{2}^{2}=10.93, P=0.004$ & \\
\hline \multicolumn{7}{|l|}{ Mother's education } \\
\hline None & 1703 & 68.5 & $6.9(5.67-8.07)$ & 1 & $14.6(12.33-15.62)$ & 1 \\
\hline Elementary school only & 440 & 17.7 & $9.5(6.31-14.08)$ & $1.430(0.989-2.069)$ & $7.5(4.72-11.68)$ & $\mathbf{0 . 4 4 9}(0.341-0.730)$ \\
\hline Up to intermediate school & 81 & 3.3 & $3.7(0.85-11.78)$ & $0.521(0.162-1.677)$ & $8.6(3.60-18.32)$ & $0.582(0.265-1.279)$ \\
\hline Up to high school & 201 & 8.1 & $3.5(2.15-5.45)$ & $\mathbf{0 . 4 8 9}(0.225-1.064)$ & $4.5(2.95-6.65)$ & $0.289(0.146-0.571)$ \\
\hline Graduate/postgraduate & 61 & 2.5 & $9.8(4.93-18.17)$ & $1.479(0.624-3.506)$ & $4.9(1.77-12.06)$ & $\mathbf{0 . 3 1 8}(0.099-1.024)$ \\
\hline Statistical test & & & $X_{1}^{2}=10.92, P=0.027$ & & $X_{4}^{2}=33.7, P<0.001$ & \\
\hline \multicolumn{7}{|l|}{ Mother's Job/profession } \\
\hline None & 2196 & 88.3 & $7.0(5.94-8.08)$ & 1 & $12.1(10.70-13.43)$ & 1 \\
\hline Blue collar worker & 218 & 8.8 & $6.0(4.10-8.53)$ & $0.841(0.469-1.508)$ & $10.1(7.58-13.19)$ & $0.818(0.517-1.294)$ \\
\hline White collar worker & 72 & 2.9 & $11.1(5.45-20.71)$ & $1.657(0.781-3.520)$ & $4.2(1.18-11.79)$ & $0.317(0.099-1.014)$ \\
\hline Statistical test & & & $\chi_{2}^{2}=0.52, P=0.77$ & & $\left.X_{2}^{2}=5.95, P<=0.051\right)$ & \\
\hline
\end{tabular}

axcluded from the analysis because sample size too small to be meaningful

${ }^{b}$ Occupation/Profession: Blue collar: mechanics, masons, builders, car wash attendants, carpenters, cleaners, crane operators, drivers, electricians, fire fighters, fitters, gardeners, labourers, painters, plumbers, steel fixers and welders; Pink collar: barbers, beauticians, butlers, grocers, hairdressers, life guards' merchandisers, nurses, safety officers/guards, sales persons, saloon workers, security guards and tailors; White collar: accountants, cashiers, civil engineers, clerks, IT experts, office boys, receptionists, and secretaries; Food handlers: bakers, butchers, chefs, cooks, kitchen assistants, waiters/waitresses; Housemaids

c This category ranged from 7 to 16 siblings

d Missing information

Note: The statistical outputs that are significant are emphasized in bolditalic, as is also the highest prevalence within each factor level

$P<0.001)$. For model 2 we fitted all significant environmental factors in Table 1 (8 factors comprising a number of rooms in the household, floor of the house, toilet, household contents index, provision of household water, farmer plus age class, and region of origin) as main effects and their 2-way interactions. Only household floor (Wald $\chi_{8}^{2}=22.5, P=0.004$ ) and household contents index (Wald $\chi_{10}^{2}=33.3, P<0.001$ ) retained significance. Region of origin of subjects was retained in the final model because Abu-Madi et al. [16] had shown this to be a significant effect on the prevalence of combined protozoan infections (Wald $\chi_{3}^{2}=8.35, P=0.039$ ), and as expected age class was also a significant factor $\left(\chi_{3}^{2}=9.12\right.$, $P=0.028$ ). Finally, in model 3 we fitted all these significant factors and their 2-way interactions. Only five factors retained significance (age class, $\chi_{3}^{2}=17.22, P=0.001$; floor of house, $\chi_{8}^{2}=19.92, P=0.011$; household contents index, $\chi_{10}^{2}=23.6, P=0.009$; father's education, $\chi_{4}^{2}=12,65$, $P=0.013$ and no of siblings, $\chi_{7}^{2}=17.34, P=0.015$ ).

Furthermore, when we fitted a log-linear model the outcome was much the same with independent effects of the principal factors fitted but with slightly different values: age (Wald $\chi_{3}^{2}=17.22, P=0.001$ ), the number of siblings (Wald $\chi_{7}^{2}=17.20, P=0.016$ ), father's education (Wald $\chi_{4}^{2}=13.75, P=0.008$ ), household floor (Wald $\left.\chi_{8}^{2}=25.20, P=0.001\right)$ and household contents index (Wald $\chi_{10}^{2}=26.73, P=0.003$ ).

\section{Discussion}

The transmission of intestinal parasites among a population is dependent firstly on the presence of infected individuals, and then for species that employ the fecal-oral route, on poor sanitation. Socioeconomic and behavioral factors in the population are also crucially important. In our study, we found that the prevalence of combined protozoan infections in the newly arrived immigrant workers to Qatar was significantly higher (12.1\%) than that among immigrant workers who had previously visited Qatar $(6.6 \%)$ and mostly had lived and worked in the city. The overall prevalence of helminth infections was lower than that of protozoan parasitic infections but the trend was in the same direction with $7.2 \%$ for the newly arrived and 
Table 2. The prevalence of helminths and protozoa in relation to environmental factors in country of origin

\begin{tabular}{|c|c|c|c|c|c|c|}
\hline & \multirow[t]{2}{*}{ N } & \multirow[t]{2}{*}{$\%$} & \multicolumn{2}{|l|}{ Combined helminths } & \multicolumn{2}{|l|}{ Combined protozoa } \\
\hline & & & Prevalence $(95 \% \mathrm{CL})$ & Odds ratio $(95 \% \mathrm{CL})$ & Prevalence $(95 \% \mathrm{CL})$ & Odds ratio $(95 \% \mathrm{CL})$ \\
\hline \multicolumn{7}{|l|}{ Ownership of home } \\
\hline Owned & 2306 & 92.7 & $7.2(6.18-8.30)$ & 1 & $18.0(16.43-19.56)$ & 1 \\
\hline Rented & 180 & 7.2 & $4.4(1.77-10.06)$ & $0.596(0.288-1.231)$ & $15.0(9.24-22.86)$ & $0.804(0.527-1.227)$ \\
\hline Statistical test & & & $\chi_{1}^{2}=2.26, P=0.77$ & & $\chi_{1}^{2}=0.24, P=0.63$ & \\
\hline \multicolumn{7}{|c|}{ No. of people living sharing house } \\
\hline 1 or 2 & 87 & 3.5 & $4.6(1.20-13.59)$ & 1 & $3.4(0.69-11.86)$ & 1 \\
\hline 3 & 398 & 16.0 & $6.5(4.08-10.24)$ & $1.450(0.493-4.267)$ & $10.6(7.25-14.93)$ & $3.303(1.000-10.915)$ \\
\hline 4 & 566 & 22.8 & $6.5(5.06-8.36)$ & $1.451(0.504-1.178)$ & $11.8(9.85-14.13)$ & $3.760(1.156-12.229)$ \\
\hline 5 & 502 & 20.2 & $6.4(4.98-8.06)$ & $1.413(0.487-4.100)$ & $13.7(11.71-16.02)$ & $4.462(1.372-14.511)$ \\
\hline 6 & 361 & 14.5 & $6.6(4.27-10.15)$ & $1.478(0.499-4.375)$ & $12.2(8.84-16.48)$ & $3.886(1.178-12.827)$ \\
\hline 7 or 8 & 347 & 13.9 & $10.1(7.08-14.04)$ & $2.328(0.804-6.735)$ & $11.8(8.57-15.97)$ & $3.752(1.134-12.417)$ \\
\hline 9 to 50 & 225 & 9.1 & $7.6(5.41-10.39)$ & $1.696(0.554-5.190)$ & $10.7(8.07-13.88)$ & $3.343(0.980-11.403)$ \\
\hline Statistical test & & & $\chi_{6}^{2}=6.19, P=0.40$ & & $\chi_{6}^{2}=10.47, P=0.11$ & \\
\hline \multicolumn{7}{|l|}{ No. of rooms in house } \\
\hline 1 & 207 & 8.3 & $6.3(4.38-8.8213)$ & 1 & $8.7(6.47-11.58)$ & 1 \\
\hline 2 & 889 & 35.7 & $7.0(5.10-9.37)$ & $1.119(0.603-2.076)$ & $11.6(9.17-14.49)$ & $1.376(0.814-2.327)$ \\
\hline 3 & 739 & 29.7 & $7.7(5.92-9.96)$ & $1.241(0.669-2.326)$ & $11.8(9.55-14.40)$ & $1.401(0.822-2.387)$ \\
\hline 4 & 407 & 16.4 & $6.9(4.32-10.71)$ & $1.102(0.558-2.177)$ & $15.2(11.26-20.22)$ & $1.887(1.084-3.283)$ \\
\hline $5-25$ & 244 & 9.8 & $6.1(4.18-8.89)$ & $0.977(0.454-2.105)$ & $8.2(5.91-11.27)$ & $0.937(0.482-1.824)$ \\
\hline Statistical test & & & $\chi_{4}^{2}=1.02, P=0.91$ & & $X_{4}^{2}=9.72, P=0.045$ & \\
\hline \multicolumn{7}{|l|}{ House construction } \\
\hline Earth and mud & 247 & 9.9 & $6.9(4.77-9.73)$ & 1 & $15.0(11.83-18.72)$ & 1 \\
\hline Wood & 385 & 15.4 & $7.3(4.70-11.05)$ & $1.061(0.568-1.982)$ & $10.1(6.97-14.34)$ & $\mathbf{0 . 6 4 0}(0.395-1.035)$ \\
\hline Bricks/stones & 782 & 31.5 & $7.7(5.83-9.99)$ & $1.124(0.643-1.966)$ & $12.9(10.53-15.73)$ & $0.842(0.550-1.265)$ \\
\hline Concrete & 1057 & 42.5 & $6.3(4.91-8.05)$ & $0.916(0.528-1.589)$ & $10.4(8.57-12.25)$ & $\mathbf{0 . 6 5 9}(0.441-0.985)$ \\
\hline Metal & 15 & 0.6 & $20(5.69-46.57)$ & $3.382(0.870-13.148)$ & $20.0(5.69-46.57)$ & $1.419(0.381-5.272)$ \\
\hline Statistical test & & & $\chi_{4}^{2}=3.99, P=0.41$ & & $\chi_{4}^{2}=7.05, P=0.13$ & \\
\hline \multicolumn{7}{|l|}{ Floor of house } \\
\hline Soil & 424 & 17.1 & $8.0(5.19-12.22)$ & 1 & $18.9(14.30-24.40)$ & 1 \\
\hline Sand & 173 & 6.9 & $6.9(3.51-13.11)$ & $0.855(0.432-1.693)$ & $7.5(3.83-13.93)$ & $0.349(0.189-0.646)$ \\
\hline Natural hard surface & 99 & 3.9 & $10.1(4.12-21.78)$ & $1.289(0.614-2.706)$ & $4.0(0.75-13.67)$ & $\mathbf{0 . 1 8 1}(0.065-0.507)$ \\
\hline Straw/other overlay & 51 & 2.1 & $7.8(3.67-15.18)$ & $0.976(0.332-2.873)$ & $9.8(5.17-17.28)$ & $0.467(0.180-1.214)$ \\
\hline Concrete/brick & 1513 & 60.9 & $6.7(5.42-7.93)$ & $0.820(0.548-1.230)$ & $11.4(9.83-13.04)$ & $\mathbf{0 . 5 5 5}(0.415-0.742)$ \\
\hline Wooden floor boards & 100 & 4.0 & $6.0(2.65-12.38)$ & $0.732(0.299-1.795)$ & $4.0(1.38-9.85)$ & $0.179(0.064-0.500)$ \\
\hline Linoleum & 62 & 2.5 & $9.7(4.80-18.08)$ & $1.229(0.494-3.059)$ & $12.9(6.94-21.86)$ & $0.637(0.292-1.392)$ \\
\hline Carpet & 6 & 0.2 & $0.0(0-41.13)$ & 0 & $0.0(0.0-41.13)$ & \\
\hline Tiles & 58 & 2.3 & $3.4(0.99-9.64)$ & $0.410(0.096-1.752)$ & $5.2(1.98-12.15)$ & $0.235(0.072-0.769)$ \\
\hline Statistical test & & & $\chi_{8}^{2}=5.26, P=0.73$ & & $X_{8}^{2}=41.10, P<0.001$ & \\
\hline \multicolumn{7}{|l|}{ Toilet } \\
\hline Flushing & 820 & 32.9 & $7.2(5.37-9.51)$ & 1 & 7.6 (5.69-9.93) & 1 \\
\hline Pit latrine & 1585 & 63.8 & $6.9(5.63-8.12)$ & $0.953(0.686-1.323)$ & $13.9(12.24-15.65)$ & $1.981(1.474-2.661)$ \\
\hline None/Bush & 81 & 3.3 & $8.6(3.60-18.32)$ & $1.220(0.538-2.768)$ & $8.6(3.60-18.32)$ & $1.156(0.511-2.618)$ \\
\hline Statistical test & & & $\chi_{2}^{2}=0.39, P=0.82$ & & $X_{2}^{2}=23.43, P<0.001$ & \\
\hline \multicolumn{7}{|c|}{ Household contents index } \\
\hline 0 & 385 & 15.5 & $9.4(6.37-13.5)$ & 1 & $16.1(12.11-20.97)$ & 1 \\
\hline 1 & 413 & 16.7 & $9.0(5.96-13.28)$ & $0.954(0.589-1.544)$ & $11.1(7.67-15.71)$ & $\mathbf{0 . 6 5 3}(0.432-0.984)$ \\
\hline 2 & 824 & 33.1 & $5.7(4.1-7.83)$ & $\mathbf{0 . 5 8 6}(0.373-0.922)$ & $14.9(12.3-17.94)$ & $0.914(0.654-1.275)$ \\
\hline 3 & 295 & 11.9 & $5.4(3.49-8.31)$ & $0.556(0.302-1.203)$ & $8.8(6.27-12.31)$ & $\mathbf{0 . 5 0 4}(0.310-0.818)$ \\
\hline
\end{tabular}


Table 2. (continued)

\begin{tabular}{|c|c|c|c|c|c|c|}
\hline & \multirow[t]{2}{*}{ N } & \multirow[t]{2}{*}{$\%$} & \multicolumn{2}{|l|}{ Combined helminths } & \multicolumn{2}{|l|}{ Combined protozoa } \\
\hline & & & Prevalence $(95 \% \mathrm{CL})$ & Odds ratio (95\% CL) & Prevalence $(95 \% \mathrm{CL})$ & Odds ratio $(95 \% \mathrm{CL})$ \\
\hline 4 & 205 & 8.2 & $7.3(5.26-9.98)$ & $0.765(0.409-1.434)$ & $4.9(3.27-7.15)$ & $\mathbf{0 . 2 6 7}(0.134-0.533)$ \\
\hline 5 & 95 & 3.8 & $8.4(3.15-19.05)$ & $0.891(0.400-1.987)$ & $6.3(1.94-16.6)$ & $0.351(0.147-0.839)$ \\
\hline 6 & 67 & 2.6 & $11.9(6.3-21.17)$ & $1.315(0.582-2.968)$ & $7.5(3.18-15.8)$ & $\mathbf{0 . 4 2 0}(0.162-1.087)$ \\
\hline 7 & 48 & 1.9 & $0.0(0-10.78)$ & - & $4.2(0.47-18.16)$ & $0.227(0.054-0.958)$ \\
\hline 8 & 55 & 2.2 & $1.8(0.29-7.26)$ & $0.180(0.024-1.337)$ & $1.8(0.29-7.26)$ & $0.096(0.013-0.710)$ \\
\hline 9 & 63 & 2.5 & $4.8(1.65-11.99)$ & $0.485(0.145-1.624)$ & $4.8(1.65-11.99)$ & $0.260(0.079-0.857)$ \\
\hline 10 & 36 & 1.4 & $11.1(4.37-24.28)$ & $1.212(0.406-3.621)$ & $16.7(7.75-31.08)$ & $1.042(0.416-2.609)$ \\
\hline Statistical test & & & $X_{1}^{2}=22.58, P=0.012$ & & $X_{1 \bar{\sigma}}^{2}=48.56, P<0.001$ & \\
\hline \multicolumn{7}{|c|}{ Provision of household water } \\
\hline Inside tap & 1329 & 53.5 & $6.8(5.45-8.32)$ & 1 & $13.6(11.78-15.46)$ & 1 \\
\hline Outside tap & 46 & 1.9 & $6.5(1.31-20.39)$ & $0.960(0.292-3.157)$ & $8.7(2.38-23.46)$ & $0.605(0.214-1.705)$ \\
\hline Shared tap & 27 & 1.1 & $3.7(0.19-18.12)$ & $0.529(0.071-3.947)$ & $0.0(0.00-12.38)$ & \\
\hline Covered well & 126 & 5.1 & $8.7(5.19-14.24)$ & $1.317(0.684-2.534)$ & $4.8(2.32-9.24)$ & $\mathbf{0 . 3 1 7}(0.138-0.731)$ \\
\hline Uncovered well & 440 & 17.7 & $8.2(5.27-12.52)$ & $1.227(0.819-1.830)$ & $13.4(9.52-18.43)$ & $0.982(0.716-1.347)$ \\
\hline Borehole & 164 & 6.6 & $6.1(2.99-11.72)$ & $0.894(0.455-1.755)$ & $9.8(5.55-16.26)$ & $0.686(0.400-1.176)$ \\
\hline River & 54 & 2.2 & $9.3(4.69-16.96)$ & $1.405(0.547-3.613)$ & $16.7(10.21-25.74)$ & $1.269(0.610-2.639)$ \\
\hline Bottled water & 300 & 12.1 & $6.3(4.18-9.42)$ & $0.931(0.558-1.552)$ & $5.0(3.15-7.82)$ & $0.334(0.194-0.575)$ \\
\hline Statistical test & & & $\chi_{7}^{2}=2.9, P=0.89$ & & $X_{7}^{2}=38.22, P<0.001$ & \\
\hline \multicolumn{7}{|c|}{ Farmer cultivate food } \\
\hline No & 1646 & 66.2 & $7.2(5.92-8.42)$ & 1 & $12.7(11.09-14.31)$ & 1 \\
\hline Yes & 840 & 33.8 & $6.8(4.99-9.08)$ & $0.943(0.679-1.308)$ & $9.6(7.49-12.28)$ & $0.734(0.559-0.962)$ \\
\hline Statistical test & & & $\chi_{1}^{2}=0.13, P=0.72$ & & $X_{1}^{2}=5.18, P=0.023$ & \\
\hline \multicolumn{7}{|c|}{ Domesticated animals } \\
\hline No & 1240 & 49.9 & $6.3(4.97-7.85)$ & 1 & $11.7(9.90-13.48)$ & 1 \\
\hline Yes & 1246 & 50.1 & $7.8(6.31-9.50)$ & $1.258(0.924-1.713)$ & $11.6(9.86-13.42)$ & $0.995(0.779-1.271)$ \\
\hline Statistical test & & & $\left(\chi_{1}^{2}=2.13, P=0.15\right)$ & & $\left(\chi_{1}^{2}=0.002, P=0.97\right)$ & \\
\hline \multicolumn{7}{|c|}{ No. of species of animals } \\
\hline 0 & 1251 & 50.3 & $6.2(4.93-7.78)$ & 1 & $11.7(9.89-13.45)$ & 1 \\
\hline 1 & 690 & 27.8 & $7.5(5.81-9.67)$ & $1.226(0.852-1.764)$ & $12.2(9.98-14.74)$ & 1.049 (0.788-1.396) \\
\hline 2 & 308 & 12.4 & $7.8(5.37-11.20)$ & $1.271(0.790-2.045)$ & $13.0(9.79-17.04)$ & $1.130(0.776-1.643)$ \\
\hline 3 & 123 & 4.9 & $9.8(5.99-15.26)$ & $1.626(0.859-3.078)$ & $6.5(3.60-11.34)$ & $\mathbf{0 . 5 2 7}(0.252-1.100)$ \\
\hline 4 & 51 & 2.1 & $13.7(8.04-22.07)$ & $2.392(0.043-5.486)$ & $13.7(8.04-22.07)$ & $0.473(0.145-1.538)$ \\
\hline 5 & 62 & 2.5 & $3.2(0.84-9.61)$ & $0.500(0.120-2.089)$ & $14.5(8.23-23.97)$ & $1.285(0.618-2.650)$ \\
\hline 6 & 1 & 0.1 & $0.0(0.00-95.0)$ & - & $0.0(0.00-95.0)$ & - \\
\hline Statistical test & & & $\chi_{6}^{2}=7.67, P=0.26$ & & $\chi_{6}^{2}=7.08, P=0.31$ & \\
\hline
\end{tabular}

Note: The statistical tests outputs that are significant are emphasized in bolditalic, as is also the highest prevalence within each factor level

4.9\% for individuals who had previously visited Qatar. Analysis by univariate statistical models of the questionnaire completed by all subjects in the study revealed that personal and familial characteristics including religion, education, number of siblings and parent's educational background, and environmental factors such as number of rooms in the house, type of floor and toilet facility, and household contents index, all played some role in influencing the prevalence of combined protozoan infection. Our data revealed also that only the mother's educational level and the household contents index had a significant effect on the prevalence of enteric helminth infections, although no clear directional trend correlating with increasing or decreasing values of the index was identified. However, fitting univariate models does not allow the influence of confounding factors and their interactions to be identified, so in the second phase of our analysis, we fitted all the significant effects from phase 1 into multifactorial models and combined these with age class and region of origin, which had been shown in our earlier 


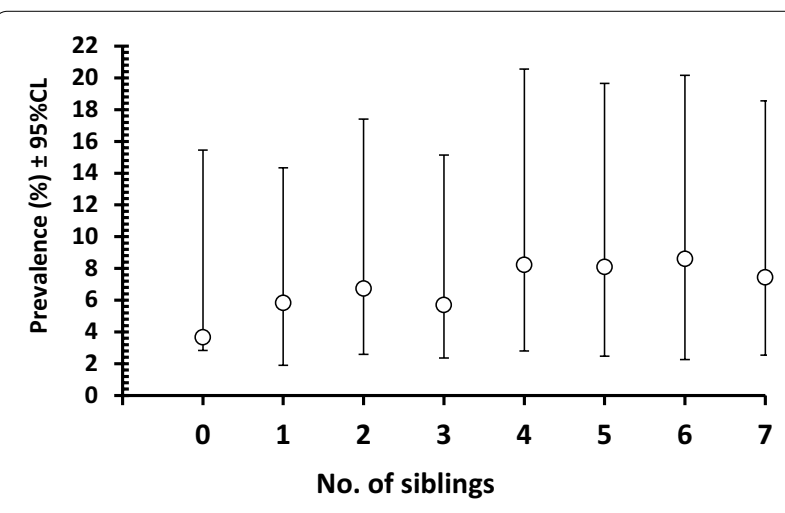

Fig. 1. Relationship between the prevalence of combined helminths and number of siblings in the family of each subject. The value of 7 siblings actually represents a range from 7 to 16. Despite the wide overlapping $95 \% \mathrm{CL}$, the correlation based on the prevalence value is significant and positive $\left(r_{s}=0.76, N=8, P=0.028\right)$

paper to have had an influence on parasitic infections in these same individuals [14]. This showed that many of the factors identified by univariate analysis are likely to have arisen through confounding interactions between the initially fitted factors. The resulting minimum sufficient models showed that the prevalence of combined helminth infections was influenced only by the host age class and the mother's educational level. The prevalence of combined protozoan infections in contrast was affected by five factors that retained significance (age class, floor of the house, household contents index, father's education, and the number of siblings).

An earlier study conducted in the Emirate of Sharjah was focused on intestinal protozoan infection rates among both immigrant workers and locals, and the infection rate here was reported as 7.7\% [17]. The prevalence of protozoan infections in our study was higher at $11.7 \%$. However, the prevalence of helminth infections in our study was marginally lower at $7.0 \%$, and lower also compared to similar studies in the region $[8,18-20]$. In addition, $17.8 \%$ of the study population carried at least one of the species (helminths + protozoa combined) that were identified.

Soil-transmitted helminth (STH) infections continue to plague large parts of the world with India known to be a significant contributor to the burden of disease [21]. STH infections are a significant health problem in Qatar given the huge number of immigrants from India and Nepal. In our earlier analyses of the prevalence of parasitic infections and their temporal trends among settled immigrants in Qatar [13], immigrants from western Asia were observed to harbor the highest prevalence of helminth infections whereas immigrants from most other regions lost their helminth burdens almost completely after acquiring residency permits. Most importantly, the prevalence of helminth infections in the period from 2005 to 2008, and then in subsequent years (2009-2011) showed a clear trend of declining prevalence in Qatar. In the current study, the lowest prevalence was observed for helminth infections among immigrants who had visited previously (4.9\%). This trend of declining prevalence of intestinal parasitic infections has been reported previously as evidence of the success of Qatar's policies [22], which demand that newcomers wishing to work and live in Qatar must undergo mandatory checks of their health in order to receive a Work Residence Permit. In addition, the efforts to introduce the usage of efficient latrines instead of open defecation, mass deworming programs, and improvements in water quality and sanitation in countries, where intestinal parasitic infections are endemic and which are the sources of the immigrant labour force in Qatar, have led to a reduction in the prevalence of these infections, as for example in India. A conducive climate for helminth transmission, rapid and unplanned urbanization, social practices of open defecation, and lack of community education and sanitation are some of the factors, which have impeded control of parasitic infections in India in the past [23]. However, India has undertaken two massive deworming programs, one starting in the year 2000 where a

Table 3. The prevalence of combined helminths infections in subjects of different age, in relation to their mother's educational level

\begin{tabular}{|c|c|c|c|c|c|c|c|c|c|c|c|c|}
\hline & \multicolumn{3}{|c|}{ Age class 1} & \multicolumn{3}{|c|}{ Age class 2} & \multicolumn{3}{|c|}{ Age class 3} & \multicolumn{3}{|c|}{ Age class 4} \\
\hline & $N$ & $\%$ & $95 \% \mathrm{CL}$ & $N$ & $\%$ & $95 \% \mathrm{CL}$ & $N$ & $\%$ & $95 \% C L$ & $N$ & $\%$ & $95 \% C L$ \\
\hline \multicolumn{13}{|l|}{ Mother's education } \\
\hline None & 215 & 10.2 & $7.72-13.33$ & 547 & 9.7 & 7.90-11.78 & 557 & 4.8 & $3.61-6.44$ & 384 & 3.9 & $2.09-6.94$ \\
\hline Elementary school & 47 & 14.9 & $5.89-31.45$ & 163 & 9.2 & $5.15-15.67$ & 154 & 7.1 & $3.80-12.94$ & 76 & 11.8 & $5.96-21.78$ \\
\hline Intermediate school & 7 & 0 & $0.00-37.71$ & 36 & 5.6 & $1.27-17.26$ & 28 & 0 & $0.00-11.94$ & 10 & 10.0 & $0.52-44.64$ \\
\hline High school & 25 & 0 & $0.00-13.36$ & 75 & 1.3 & $0.12-7.79$ & 71 & 5.6 & $2.02-13.74$ & 30 & 6.7 & $1.20-21.34$ \\
\hline Tertiary level & 9 & 22.2 & $4.11-55.82$ & 35 & 2.9 & $0.32-13.16$ & 13 & 15.4 & $2.81-43.39$ & 4 & 25.0 & $1.28-75.13$ \\
\hline
\end{tabular}

Note: The statistical tests outputs that are significant are emphasized in bolditalic 
single dose of Albendazole and DEC was administered to communities in the filarial-endemic regions and another in 2015 covering 241 million children for the treatment of STH infections [24, 25]. These have been very successful in reducing the prevalence of helminth infections in the country $[24,25]$.

In the present study, a relatively high prevalence of protozoan parasitic infections (15\%) was initially found in univariate models to be associated with the Hindu religion, but the influence of religion was not retained in models that took into account other factors and is likely therefore to be a consequence of the confounding effect of other markers that reflect the subjects living conditions in their country of origin. Our finding might be due to the fact that the Hindu community are composed of Indian and Nepalese nationals [26], among whom protozoan infections are highly endemic. On the other hand, no significant difference in the prevalence of helminth infections was observed between subjects practicing different religions, which may be due to massive deworming programs conducted in endemic countries.

In our study, we observed that the individual's educational level and that of their parents also had an important influence on the prevalence of protozoan infections. Prevalence was highest among uneducated subjects $(15.2 \%)$ and also among those whose parents were illiterate $(14.6 \%$ in both cases) and this was a highly significant finding. There was also a trend of decreasing prevalence with increasing level of education. Other studies have shown also that a mother's literacy is an important socio-economic factor influencing parasite prevalence [27-30]. Another study has reported similar results to our study [31], with increasing parent's educational level correlating with the declining prevalence of protozoan infections. We found a similar trend when examining the parents' occupational levels, the prevalence of protozoan infections declining consistently with increasing father's occupational level from no occupation (12.6\%), blue collar workers (11.9\%), and then white collar workers (5.3\%). A similar continuous reduction in prevalence was observed also with the mother's occupational level from no occupation (12.1\%) to white collar workers (4.2\%). In contrast, our analysis found no significant effects of occupational level on the prevalence of helminth infections, although there was a somewhat surprising finding in relation to the mother's educational level, but this did not change consistently with increasing level of education. The highest prevalence of helminth infections was among the offspring of graduates.

In our analysis of the influence of environmental factors in the country of origin on protozoan and helminth infections, we observed that in general large families were more prone to infection. Although across the seven levels of house occupants detailed in Table 1, there were no significant differences, it is nevertheless interesting to note that helminth and protozoan infections were least prevalent among people living alone or in couples. Notably, the prevalence of protozoan infections increased from just 3.4\% among people living alone or in couples, to over $10 \%$ in all other cases and a maximum of $13.7 \%$ in the case of 5 occupants in a household. Our results are consistent with Halpenny et al. [32] who found that the large families (with more than three children) were more likely to experience a high prevalence of intestinal parasitic infections and higher co-infection patterns with multiple species, and these are likely to be attributable to overcrowding conditions in households [32]. In addition, we found the highest prevalence of protozoan infections among people who lived in houses with only soil as the floor (18.9\%). Considering other possible household deficiencies that may enhance transmission of parasites between household inhabitants, and hence lead to higher prevalence, water and sanitization are two such key components. Access to clean water and efficient sanitary facilities within or in proximity to the household are essential to prevent deleterious effects on the health of inhabitants. In our study, the prevalence of protozoan infections was highest among individuals whose only supply of drinking water was directly from a local river (16.7\%), or who exploited water from an uncovered well (13.4\%). However, perhaps unexpectedly, even those who had access to a tap indoors, were also subject to a relatively high risk of protozoan infection, in this case being $13.6 \%$, which indicates perhaps that the water supplies in these countries are contaminated. Interestingly, those who relied primarily on bottled water and/or used a covered well were less likely to be infected. The prevalence of helminth infections was also relatively high among individuals drinking river water (9.3\%).

The prevalence and control of STH and protozoan infections are inextricably linked to water quality, sanitation, hygiene practices and the socio-economic status of communities in regions where these parasites are endemic [33]. Studies have shown that improved water quality, efficient sanitary facilities, and good hygiene practice, all contribute significantly to preventing diarrhea, morbidity, and mortality caused by protozoa and soil transmitted helminth in low- and middle-income countries [34]. Therefore, household access to clean tap water, safe disposal of excreta (for example use of flushing toilets instead of open defecation) and education about good hygiene practice are crucially important for targeted interventions aimed at reducing the incidence of intestinal parasitic infections [33, 35, 36]. The vulnerability of drinking water supply systems to contamination by pathogens and the consequent increase of risk of waterborne diseases have been highlighted in several 
studies [37, 38]. In addition, the protection of drinking water from these protozoa is a serious problem for water supply organizations around the world. Cryptosporidium and Giardia remain the two most important water pathogens that could not be eradicated until relatively recently [34]. Giardia is an anaerobic flagellated protozoa capable of encysting through a complex process of cyst wall formation [39], with this infective form being resistant to common disinfection controls such as chlorine and chloramines [40].

Since the intestinal helminths and protozoa studied in the current work are all dependent on fecal-oral transmission, the proper, safe and efficient management of feces and its disposal are key issues. When the surrounding environment is contaminated with feces, the magnitude of the problem may seem overwhelming [41-44]. Pit latrines are often recommended as an important step away from open defecation in the bush, but in our study, we observed that $13.9 \%$ of individuals who use pit latrines in their home country suffered from protozoan infection, a figure that is significantly higher than the prevalence among those using flushing toilets, and even open defecation. Throughout the world, there is considerable variation in the use of different types of toilets. Approximately 1.77 billion people around the world use pit latrines as the primary means of sanitation. Pit latrines are the simplest and most inexpensive form of improved sanitation, but they have to be maintained carefully to avoid infections. Pit latrines usually lack a physical barrier, such as concrete, between stored excrement and soil and/or groundwater [45]. In some countries where pit latrines are common, more than two billion people use groundwater as a source of drinking water [45]. Therefore, contaminants from pit latrines can also enter groundwater and create a threat to human health.

Our study is the first comprehensive study to address the issue of parasitic prevalence in an apparently healthy immigrant population in Qatar. However, our study suffered from certain limitations. First, laboratory diagnosis of intestinal parasitic infection (IPI) was based on a single stool examination, which could have underestimated the prevalence, as optimal laboratory diagnosis of IPIs requires the examination of at least three stool specimens collected over several days [46], but clearly this was just not possible in our study. However, more recent studies have suggested that one or two stool samples will detect up to $90 \%$ of the protozoa present $[47,48]$.

\section{Conclusions}

The increased prevalence of protozoan infections among migrant workers in Qatar over recent years [13] raises some concerns. In contrast to the helminth infections which as adjudged by the current data, appear to be increasingly well-controlled among immigrants prior to their arrival in Qatar, protozoan infections among new arrivals appear to be increasing, at least in the short-term. Our work provides useful benchmark information for prioritizing interventions in their counties of origin. In addition, it emphasizes the importance of regular checks for intestinal protozoan infections and subsequent treatment with anti-protozoan agents prior to arrival in Qatar. We believe that this will be a highly desirable course of action for the future, and we strongly recommend that Qatar's health authorities implement such measures in the near future.

\section{Abbreviations}

CL: Confidence limits; rpm: Revolutions per minute; GLMs: Generalized linear models; STH: Soil-transmitted helminth; IPI: Intestinal parasitic infection.

\section{Supplementary information}

Supplementary information accompanies this paper at https://doi.org/10. 1186/s13071-020-04449-9.

Additional file 1: Table S1. Prevalence of intestinal helminths and protozoan parasites in the study group. Data from [14], showing the values for prevalence of each of the seven species of helminths, eight species of protozoan parasites, overall prevalence of helminths, protozoa and both groups combined

\section{Acknowledgements}

We would like to thank all the technical staff of the Medical Commission, Ministry of Public Health and to the Biomedical Research Center at Qatar University for providing the facilities for this work.

\section{Disclaimer}

The contents of this report are solely the responsibility of the authors and do not necessarily represent the official views of Qatar University and Qatar National Research Fund.

\section{Authors' contributions}

Conceptualization: Al and MAM. Data curation: JMB and MAM. Formal analysis: NY and JMB. Funding acquisition: MAM. Investigation: MAM and AI. Methodology: NY, JMB and MAM. Project administration: Al and MAM. Resources: NY and MAM. Software: MAM and JMB. Supervision: Al and MAM. Validation: JMB and MAM. Visualization: NY and JMB. Writing (original draft): NY, JMB and MAM. Writing (review and editing): MAM and JMB. All authors read and approved the final manuscript.

\section{Funding}

This publication was made possible by a National Priority Research Program grant number NPRP8-1556-3-313 from the Qatar National Research Fund (https://www.qnrf.org/), a member of Qatar Foundation.

\section{Availability of data and materials Not applicable.}

\section{Ethics approval and consent to participate}

Ethical approval to undertake the study in the current design was obtained from the Medical Research Centre and Research Committee at HMC, Qatar (Research protocol \# 16367/16 (NPRP8-1556-3-313). Additionally, written informed consent was obtained from each participant on a document, that included an explanation of the significance of the study, participant requirements and rights, and information on sample collection. Confidentiality 
was maintained throughout and the identity of subjects was not available to us, other than through each individual's reference number. Age, sex, and geographical region were recorded for each patient before taking a stool specimen.

\section{Consent for publication}

Not applicable.

\section{Competing interests}

The authors declare that they have no competing interests.

\section{Author details}

${ }^{1}$ Biomedical Science Department, College of Health Sciences, Biomedical Research Center, Qatar University, P.O. Box 2713, Doha, Qatar. ${ }^{2}$ School of Life Sciences, University of Nottingham, University Park, Nottingham NG7 2RD, UK. ${ }^{3}$ Medical Commission, Ministry of Public Health, P.O. Box 42, Doha, Qatar.

Received: 14 July 2020 Accepted: 30 October 2020

Published online: 20 January 2021

\section{References}

1. Brooker S, Clements AC, Bundy DA. Global epidemiology, ecology and control of soil-transmitted helminth infections. Adv Parasitol. 2006:62:221-61.

2. Dachs JN, Ferrer M, Florez CE, Barros AJ, Narvaez R, Valdivia M. Inequalities in health in Latin America and the Caribbean: descriptive and exploratory results for self-reported health problems and health care in twelve countries. Rev Panam Salud Publica. 2002;11:335-55.

3. Savioli L, Albonico M, Engels D, Montresor A. Progress in the prevention and control of schistosomiasis and soil-transmitted helminthiasis. Parasitol Int. 2004;53:103-13.

4. Celiksoz A, Guler N, Guler G, Oztop AY, Degerli S. Prevalence of intestinal parasites in three socioeconomically-different regions of Sivas, Turkey. J Health Popul Nutr. 2005;23:184-91.

5. Woh PY, Thong KL, Behnke JM, Lewis JW, Mohd Zain SN. Evaluation of basic knowledge on food safety and food handling practices amongst migrant food handlers in Peninsular Malaysia. Food Control. 2016;70:64-73.

6. Sahimin N, Lim YA, Ariffin F, Behnke JM, Lewis JW, Mohd Zain SN. Migrant workers in Malaysia: current implications of sociodemographic and environmental characteristics in the transmission of intestinal parasitic infections. PLoS Negl Trop Dis. 2016;10:e0005110.

7. Andargie G, Kassu A, Moges F, Tiruneh M, Huruy K. Prevalence of bacteria and intestinal parasites among food-handlers in Gondar town, northwest Ethiopia. J Health Popul Nutr. 2008;26:451-5.

8. Sharif M, Daryani A, Kia E, Rezaei F, Nasiri M, Nasrolahei M. Prevalence of intestinal parasites among food handlers of Sari, northern Iran. Rev Inst Med Trop Sao Paulo. 2015;57:139-44.

9. Wang LC. Changing patterns in intestinal parasitic infections among Southeast Asian laborers in Taiwan. Parasitol Res. 2004;92:18-21.

10. Zaglool DA, Khodari YA, Othman RAM, Farooq MU. Prevalence of intestinal parasites and bacteria among food handlers in a tertiary care hospital. Niger Med J. 2011;52:266-70.

11. Takalkar AA, Madhekar NS, Kumavat AP, Bhayya SM. Prevalence of intestinal parasitic infections amongst food handlers in hotels and restaurants in Solapur city. Indian J Public Health. 2010;54:47-8.

12. Kheirandish F, Tarahi MJ, Ezatpour B. Prevalence of intestinal parasites among food handlers in Western Iran. Rev Inst Med Trop Sao Paulo. 2014;56:111-4.

13. Abu-Madi MA, Behnke JM, Doiphode SH. Intestinal parasitic infections among long-term-residents and settled immigrants in Qatar in the period 2005 to 2011. Am JTrop Med Hyg. 2013;88:1185-95.

14. Abu-Madi MA, Behnke JM, Ismail A, Boughattas S. Assessing the burden of intestinal parasites affecting newly arrived immigrants in Qatar. Parasites Vectors. 2016;9:619.

15. Abu-Madi M, Boughattas S, Behnke JM, Sharma A, Ismail A. Coproscopy and molecular screening for detection of intestinal protozoa. Parasites Vectors. 2017;10:414.
16. Abu-Madi M, Behnke JM, Sharma A, Bearden R, Al-Banna N. Prevalence of virulence/stress genes in Campylobacter jejuni from chicken meat sold in Qatari retail outlets. PLoS ONE. 2016;11:e0156938.

17. Dafalla AIA, Almuhairi SASO, AlHosani MHJ, Mohamed MY, Alkous MIA, AlAzzawi MA, et al. Intestinal parasitic infections among expatriate workers in various occupations in Sharjah, United Arab Emirates. Rev Inst Med Trop Sao Paulo. 2017;59:e82.

18. Abahussain N. Prevalence of intestinal parasites among expatriate workers in Al-Khobar, Saudi Arabia. Middle East J Fam Med. 2005;3:17-21.

19. Taha HA, Soliman MI, Banjar SA. Intestinal parasitic infections among expatriate workers in Al-Madina Al-Munawarah, Kingdom of Saudi Arabia. Trop Biomed. 2013;30:78-88.

20. Astal Z. Epidemiological survey of the prevalence of parasites among children in Khan Younis governorate, Palestine. Parasitol Res. 2004;94:449-51.

21. Lobo DA, Velayudhan R, Chatterjee P, Kohli H, Hotez PJ. The neglected tropical diseases of India and South Asia: review of their prevalence, distribution, and control or elimination. PLoS Negl Trop Dis. 2011;5:e1222.

22. Government Qe. Medical Commission Procedures.

23. Salam N, Azam S. Prevalence and distribution of soil-transmitted helminth infections in India. Infect Dis Poverty. 2017;17:201.

24. Awasthi S, Peto R, Read S, Richards SM, Pande V, Bundy D, et al. Population deworming every 6 months with albendazole in 1 million pre-school children in North India: DEVTA, a cluster-randomised trial. Lancet. 2013;381:1478-86.

25. Ganguly S, Barkataki S, Karmakar S, Sanga P, Boopathi K, Kanagasabai K, et al. High prevalence of soil-transmitted helminth infections among primary school children, Uttar Pradesh, India, 2015. Infect Dis Poverty. 2017;6:139.

26. Hotez PJ. The world's great religions and their neglected tropical diseases. PLoS Negl Trop Dis. 2016;10:e0004544.

27. Mamatha G, Munirathnamma K. Awareness of mothers of under five year children regarding round worm infestation, its prevention and management: a descriptive analysis. Int J Nurs Educ. 2014;6(1):179.

28. Zonta ML, Oyhenart EE, Navone GT. Socio-environmental variables associated with malnutrition and intestinal parasitoses in the child population of Misiones, Argentina. Am J Hum Biol. 2014;26:609-16.

29. Buor D. Mothers' education and childhood mortality in Ghana. Health Policy. 2003;64:297-309.

30. Quihui L, Valencia ME, Crompton DWT, Phillips S, Hagan P, Morales G, et al. Role of the employment status and education of mothers in the prevalence of intestinal parasitic infections in Mexican rural schoolchildren. BMC Public Health. 2006;6:225.

31. Bahmani P, Maleki A, Sadeghi S, Shahmoradi B, Ghahremani E. Prevalence of intestinal protozoa infections and associated risk factors among schoolchildren in Sanandaj City, Iran. Iran J Parasitol. 2017;12:108-16.

32. Halpenny CM, Koski KG, Valdes VE, Scott ME. Prediction of child health by household density and asset-based indices in impoverished indigenous villages in rural Panama. Am J Trop Med Hyg. 2012;86:280-91.

33. Strunz EC, Addiss DG, Stocks ME, Ogden S, Utzinger J, Freeman MC. Water sanitation, hygiene, and soil-transmitted helminth infection: a systematic review and meta-analysis. PLoS Med. 2014;11:e1001620.

34. Omarova A, Tussupova K, Berndtsson R, Kalishev M, Sharapatova K. Protozoan parasites in drinking water: a system approach for improved water, sanitation and hygiene in developing countries. Int J Environ Res Public Health. 2018;15:495.

35. Ziegelbauer K, Speich B, Mausezahl D, Bos R, Keiser J, Utzinger J. Effect of sanitation on soil-transmitted helminth infection: systematic review and meta-analysis. PLoS Med. 2012;9:e1001162.

36. Boisson S, Sosai P, Ray S, Routray P, Torondel B, Schmidt WP, et al. Promoting latrine construction and use in rural villages practicing open defecation: process evaluation in connection with a randomised controlled trial in Orissa, India. BMC Res Notes. 2014;7:486.

37. Nescerecka A, Rubulis J, Vital M, Juhna T, Hammes F. Biological instability in a chlorinated drinking water distribution network. PLoS ONE. 2014:9:e96354.

38. Rodriguez-Alvarez MS, Weir MH, Pope JM, Seghezzo L, Rajal VB, Salusso $\mathrm{MM}$, et al. Development of a relative risk model for drinking water regulation and design recommendations for a peri urban region of Argentina. Int J Hyg Environ Health. 2015;218:627-38. 
39. Rivero MR, Vranych CV, Bisbal M, Maletto BA, Ropolo AS, Touz MC. Adaptor protein 2 regulates receptor-mediated endocytosis and cyst formation in Giardia lamblia. Biochem J. 2010;428:33-45.

40. Betancourt WQ, Rose JB. Drinking water treatment processes for removal of Cryptosporidium and Giardia. Vet Parasitol. 2004;126:219-34.

41. Caccio SM, De Giacomo M, Aulicino FA, Pozio E. Giardia cysts in wastewater treatment plants in Italy. Appl Environ Microbiol. 2003;69:3393-8.

42. Fayer R. Cryptosporidium: a water-borne zoonotic parasite. Vet Parasitol. 2004:126:37-45

43. Montemayor M, Valero F, Jofre J, Lucena F. Occurrence of Cryptosporidium spp. oocysts in raw and treated sewage and river water in north-eastern Spain. J Appl Microbiol. 2005;99:1455-62

44. Ruecker NJ, Braithwaite SL, Topp E, Edge T, Lapen DR, Wilkes G, et al. Tracking host sources of Cryptosporidium spp. in raw water for improved health risk assessment. Appl Environ Microbiol. 2007;73:3945-57.
45. Graham JP, Polizzotto ML. Pit latrines and their impacts on groundwater quality: a systematic review. Environ Health Perspect. 2013;121:521-30.

46. Branda JA, Lin TY, Rosenberg ES, Halpern EF, Ferraro MJ. A rational approach to the stool ova and parasite examination. Clin Infect Dis. 2006;42:972-8.

47. Haque R. Human intestinal parasites. J Health Popul Nutr. 2007;25:387-91.

48. Marti H, Koella JC. Multiple stool examinations for ova and parasites and rate of false-negative results. J Clin Microbiol. 1993;31:3044-5.

\section{Publisher's Note}

Springer Nature remains neutral with regard to jurisdictional claims in published maps and institutional affiliations.
Ready to submit your research? Choose BMC and benefit from:

- fast, convenient online submission

- thorough peer review by experienced researchers in your field

- rapid publication on acceptance

- support for research data, including large and complex data types

- gold Open Access which fosters wider collaboration and increased citations

- maximum visibility for your research: over $100 \mathrm{M}$ website views per year

At BMC, research is always in progress.

Learn more biomedcentral.com/submissions 